\title{
Dilute Russel Viper Venom Time analysis in a Haematology Laboratory: an audit report
}

Welma Kruger ${ }^{1}$, Pieter Meyer ${ }^{2}$, Jan Gert Nel ${ }^{1,{ }^{*}}$

${ }^{1}$ Department of Haematology, Faculty of Health Sciences, NHLS - Tshwane Academic Division, Institute of Pathology, University of Pretoria, Riviera, Pretoria, South Africa

${ }^{2}$ Department of Immunology, Faculty of Health Sciences, NHLS - Tshwane Academic Division, Institute of Pathology, University of Pretoria, Riviera, Pretoria, South Africa

${ }^{*}$ Correspondence to:

Dr JG Nel

jan.nel@up.ac.za

Department of Haematology

Institute of Pathology room 2-66

Faculty of Health Sciences, University of Pretoria Prinshof campus

5 Bophelo Road (c/o Steve Biko Rd \& Dr Savage Rd)

Riviera, Pretoria

South Africa

0154 


\section{ABSTRACT}

Aim

To determine if the current set of evaluation criteria used for dilute Russel Viper Venom Time (dRVVT) investigations in the routine laboratory meet expectation and identify possible shortcomings.

\section{Methods}

All dRVVT assays requested from January 2015 to December 2015, were appraised in this crosssectional study. The raw data panels were compared to the new reference interval, established in 2016, to determine the sequence of assays that should have been performed. The interpretive comments were audited, and false-negative reports identified. Interpretive comments according to three interpretation guidelines were compared. The reagent cost per assay was determined and reagent cost wastage, due to redundant tests, was calculated.

\section{Results}

Only $\sim 9 \%$ of dRVVT results authorized during 2015 , had an interpretive comment included in the report. $\sim 15 \%$ of these results were false negative interpretations. There is a significant statistical difference in interpretive comments between the three interpretation methods. Redundant mixing tests resulted in $\mathrm{R} 7477.91$ ( 11\%) reagent cost wastage in 2015.

\section{Conclusions}

We managed to demonstrate very evident deficiencies in our own practice and managed to establish a standardised workflow that will potentially render our service more efficient and cost effective, aiding clinicians in making improved treatment decisions and diagnoses. Furthermore, it is essential that standard operating procedures be kept up-to-date and executed by all staff in the laboratory. 


\section{INTRODUCTION}

Antiphospholipid syndrome (APS) is an autoimmune disease with two main characteristics, the presence of persistent antiphospholipid antibodies (aPLs) and clinical features including thrombosis and pregnancy complications. International experts developed the Sapporo Criteria in 1999 to aid in the classification of APS which was updated in 2006..$^{1,2}$ The diagnosis of APS requires at least one clinical and one laboratory criterion. This study focused on the interpretation of dilute Russel Viper Venom Time (dRVVT) results and the communication of diagnostic findings.

Assays used for LA detection are a combination of screening and confirmatory tests. Dilute Russell Viper Venom Time and activated partial thromboplastin time (aPTT) are the most common combination of screening tests used. Test results for LA determination are prone to variability and false positivity, necessitating repeat testing to confirm positivity. ${ }^{3}$

A comprehensive analysis of the interpretation of dRVVT was undertaken in our laboratory, as the challenges associated with this test are well documented. Our aim was to determine adherence to standard operating procedures (SOPs) and highlight any deviations that may have existed.

Authoritative guidelines available for the testing of LA, include the International Society on Haemostasis and Thrombosis 2009 guidelines. ${ }^{4}$ the British Committee for Standards in Haematology 2012 guidelines and the Clinical and Laboratory Standards Institute 2014 guidelines. ${ }^{5}$ All guidelines advise the use of normalized ratios (NR), as this reduce the effects of inter-and intra-assay variation. ${ }^{5-8}$ ISTH 2009 and BSCH 2012 suggests using the normal pooled plasma (NPP) value as denominator in determining ratios. ${ }^{6-8}$ CLSI 2014 recommends using the mean clotting time of the reference interval $(\mathrm{RI})$ for a specific assay as the denominator in calculating ratios. The rationale for using RI mean as denominator is because of inter-batch differences in NPP time, for example the variability encountered between lyophilized NPP and fresh NPP batches. It also compensates for inter- and intra-assay variability (operator/reagent/analyser), as these should have been considered in establishing the RI. All three guidelines recommend the establishment of laboratory specific reference intervals. CLSI 2014 emphasizes that the RI needs to be verified with each new lot of reagents when the $\mathrm{RI}$ mean is used as denominator in the normalized ratio, otherwise compensation for day-to-day variation is lost. The guideline also specifically states that normalization to the mean of the RI should only be done if in-line with manufacturers' instructions. ${ }^{5}$

Although the advantages of having locally developed cut-off values has been stated by Tripodi et al. ${ }^{9}$, the financial and other logistical implications of introducing it in our local practice was and still remains a major obstacle. Therefore, the "next best" alternative, of utilising cut-off values as determined elsewhere, was employed. A 'universal' cut-off ratio, for the interpretation of dRVVT $L A$ panels, as was established by using data from the Royal College of Pathologists of Australasia 
(RCPA) Quality Assurance Program (QAP) was chosen. Most laboratories reported a positive dRVVT finding for dRVVT normalized ratios, against NPP, above 1.2. ${ }^{10}$

\section{MATERIALS AND METHODS}

Our laboratory uses LA sensitive aPTT and dRVVT as screening investigations where a clinician suspects the presence of LA. For the purpose of this study, only dRVVT was assessed.

Verification and implementation of reference ranges were inadequate with the change of coagulation analyser to the Siemens CS2100i, and reagents to Siemens LA1 Screening Reagent and LA2 Confirmation Reagent ( $L A 1=$ screening reagent for $\mathrm{dRVVT}$, $L A 2=$ confirming dRVVT reagent). Both these reagents are dRVVT assays, with LA2 having a higher concentration of phospholipids. A new reference interval was established early in 2016, by running specimens obtained from 40 healthy donors. These results were compared to the reference intervals suggested by the manufacturer of the reagents. CLSI 2014 guidelines and guidelines by Siemens for the use of the Siemens CS2100i analyser and reagents were employed. The reference interval, for LA1 screen dRVVT, subsequently changed from 31.89-48.05 seconds to 33.80-43.90 seconds. The reference interval, LA2 confirm dRVVT remained the same, 28.63-36.55 seconds.

Data from all dRVVT assays performed from January 2015 to December 2015 were examined in a cross-sectional study. Due to the unavailability of the newer anticoagulants in public health care in our setting, none of the patients, to our knowledge, in this study was on any of the new oral anticoagulants.

\section{Setting}

The National Health Laboratory Service Tshwane Academic Division (NHLS TAD) Haematology Laboratory, is based at Steve Biko Academic Hospital, Pretoria, South Africa. This laboratory serves as a reference laboratory for LA determination, for several other NHLS laboratories based in Gauteng, Mpumalanga, Limpopo and North West provinces.

\section{Data collection}

The raw data of all $L A d R V V T$ assays, performed from January 2015 to December 2015, were captured in a Microsoft Excel spreadsheet. The interpretive report for each LA panel, authorized on the Laboratory Information Service (LIS), was also noted. 


\section{Data processing}

Challenges identified during raw data capturing include:

- Very few dRVVT panels contained an interpretive comment.

- Discrepancies in interpretation of raw data results. The following two methods were used to interpret results:

1. The raw result (in seconds) was compared to the $\mathrm{RI}$, and decision to continue to the next step was based on the interpretation as illustrated in table 1. The proposed interpretation for each panel is also indicated in table 1.

\begin{tabular}{|c|c|c|c|c|}
\hline \multicolumn{2}{|c|}{ Patient Plasma } & \multicolumn{2}{|c|}{$\begin{array}{c}\text { Mixing studies } \\
\text { (Patient plasma:NPP = 1:1) }\end{array}$} & \multirow{2}{*}{$\begin{array}{l}\text { Proposed interpretation and } \\
\text { comment }\end{array}$} \\
\hline $\begin{array}{l}\text { LA ratio } \\
\text { (LA1/NPP) }\end{array}$ & $\begin{array}{l}\text { LA2 ratio } \\
\text { (LA2/NPP) }\end{array}$ & $\begin{array}{l}\text { LA1 mix ratio } \\
\text { (LA1 mix/NPP) }\end{array}$ & $\begin{array}{l}\text { LA2 mix ratio } \\
\text { (LA2 mix/NPP) }\end{array}$ & \\
\hline $\begin{array}{l}<1.2-\text { stop } \\
\text { testing }\end{array}$ & - & - & - & LA not detected \\
\hline$\geq 1.2$ & $\begin{array}{c}<1.2-\text { stop } \\
\text { testing \& } \\
\text { calculate } N R^{* \star}\end{array}$ & - & - & If $N R \geq 1.2=$ LA present \\
\hline$\geq 1.2$ & $\geq 1.2$ & $\begin{array}{l}<1.2-\text { stop } \\
\text { testing }\end{array}$ & - & $\begin{array}{l}\text { LA not detected; factor deficiency, } \\
\text { or vitamin K antagonist therapy }\end{array}$ \\
\hline$\geq 1.2$ & $\geq 1.2$ & $\geq 1.2$ & $\begin{array}{c}<1.2-\text { calculate } \\
\quad N R \text { mix }^{* * *}\end{array}$ & $\begin{array}{l}\text { If NR mix } \geq 1.2 \text { = LA present; } \\
\text { concomitant factor deficiency, or } \\
\text { vitamin } \mathrm{K} \text { antagonist therapy }\end{array}$ \\
\hline$\geq 1.2$ & $\geq 1.2$ & $\geq 1.2$ & $\geq 1.2$ & $\begin{array}{l}\text { Indeterminate, probably another } \\
\text { inhibitor or strong LA }\end{array}$ \\
\hline \multicolumn{5}{|c|}{$\begin{array}{l}{ }^{*} \mathrm{~N}=\text { Normal } \\
{ }^{* *} \mathrm{NR}=\text { normalized ratio (LA1 ratio/LA2 ratio) } \\
{ }^{* * *} \mathrm{NR} \text { mix = LA1 mix ratio/LA2 mix ratio }\end{array}$} \\
\hline
\end{tabular}

2. Calculating the screen/NPP (LA1 ratio) or confirm/NPP (LA2 ratio) ratio for each assay and comparing the LA1 and LA2 ratios to the universal cut-off of 1.2 to determine whether to proceed to the next step or not (refer to figure 1). The normalised ratio (LA1ratio/LA2 ratio) was used to interpret LA panels and establish an interpretive comment, using table 1 as guideline. 


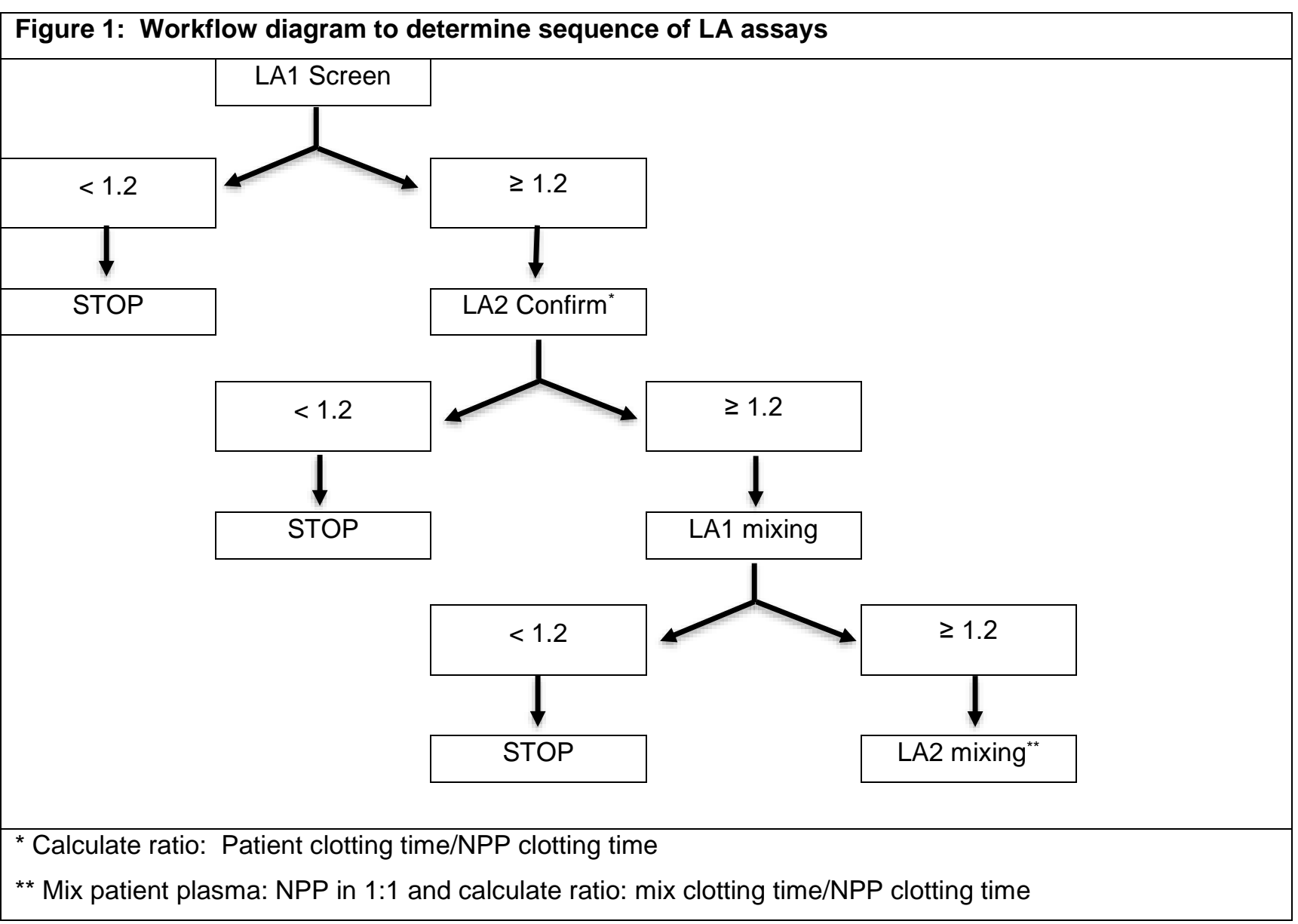

The raw data were subsequently re-interpreted, generating a new interpretive comment. The following methods were used to interpret each dRVVT panel:

1. The clotting time (in seconds) was compared to the new reference intervals for clotting times (in seconds).

2. LA1 and LA2 ratios, using NPP as denominator, were calculated for each assay. These ratios were evaluated using $\geq 1.2$ as abnormal. The normalised ratio was determined if indicated as per figure 1 , and an interpretive comment generated.

3. LA1 and LA2 ratios, using the RI mean, were calculated for each assay. These ratios were evaluated using $\geq 1.2$ as abnormal. The normalised ratio was determined if indicated as per figure 1, and an interpretive comment generated using table 1.

The interpretive comments for all three methods were compared.

\section{Statistical analysis}

All analyses were performed using Microsoft Excel for Windows and STATA version 13.1 statistical software.

\section{RESULTS}

A total of 2273 patient samples were processed for dRVVT determination, from January 2015 to December 2015, in the NHLS TAD Haematology Laboratory. 
Only 208 (9\%) of the 2273 dRVVT panels had an authorized comment. 90 (43\%) of the 208 DRVVT data sets had a standard comment, but no interpretation, added to the report.

All dRVVT panels were re-interpreted, using the patient clotting time/NPP clotting time ratio. This ratio was compared to the universal cut-off ratio of $\geq 1.2$ as abnormal, as was stated in the SOP. 31 (15\%) of the 208 panels were false negative after re-classification. The relative reports were amended, stating the reason for the amendment. The requesting clinician was also contacted (if reachable) and notified of the amended report.

478 (21\%) of the 2273 dRVVT panels should have had an interpretive comment added, but this was omitted from the report. This is in direct conflict with all available guidelines for dRVVT determination. These reports were reviewed, and an interpretive comment added.

The remaining $1588(70 \%)$ of the 2273 dRVVT panels only had a LA1 Screening clotting time/LA1 NPP clotting time ratio, which were $<1.2$, and easily interpreted as "LA not detected".

The comparisons of the interpretive comments, using the three methods described above, are demonstrated in table 2 and 3.

\begin{tabular}{|c|c|c|c|c|c|}
\hline & & \multicolumn{3}{|c|}{ NPP denominator } & \\
\hline & & $L A$ detected ${ }^{*}$ & $\begin{array}{c}\text { No } L A \\
\text { detected }^{* *}\end{array}$ & Uninterpretable*** & \\
\hline \multirow{4}{*}{$\begin{array}{l}\text { Clotting time } \\
\text { (in seconds) }\end{array}$} & LA detected $^{*}$ & 31 & 13 & 0 & 43 \\
\hline & $\begin{array}{c}\text { No } L A \\
\text { detected }^{* *}\end{array}$ & 9 & 1451 & 0 & 1460 \\
\hline & $\underset{* * *}{\text { Uninterpretable }}$ & 83 & 504 & 184 & 771 \\
\hline & & 123 & 1967 & 184 & 2274 \\
\hline \multicolumn{6}{|c|}{$\begin{array}{l}\text { *: Include all reports with LA detected, with/without factor deficiency or vitamin K anticoagulant therapy } \\
\text { (VKA) } \\
\text { **: Include all reports with no LA detected, with/without factor deficiency or vitamin K anticoagulant therapy } \\
\text { (VKA) } \\
\text { ***: Include all reports that required further testing to clarify the pattern of results. Samples were either } \\
\text { insufficient to perform further testing, or the raw result in seconds was within the normal reference interval } \\
\text { and testing was stopped, even though the ratio (LA1 or LA2 ratio) was } \geq 1.2 \text {. } \\
\text { NPP: Normal pooled plasma; LA: Lupus Anticoagulant }\end{array}$} \\
\hline
\end{tabular}




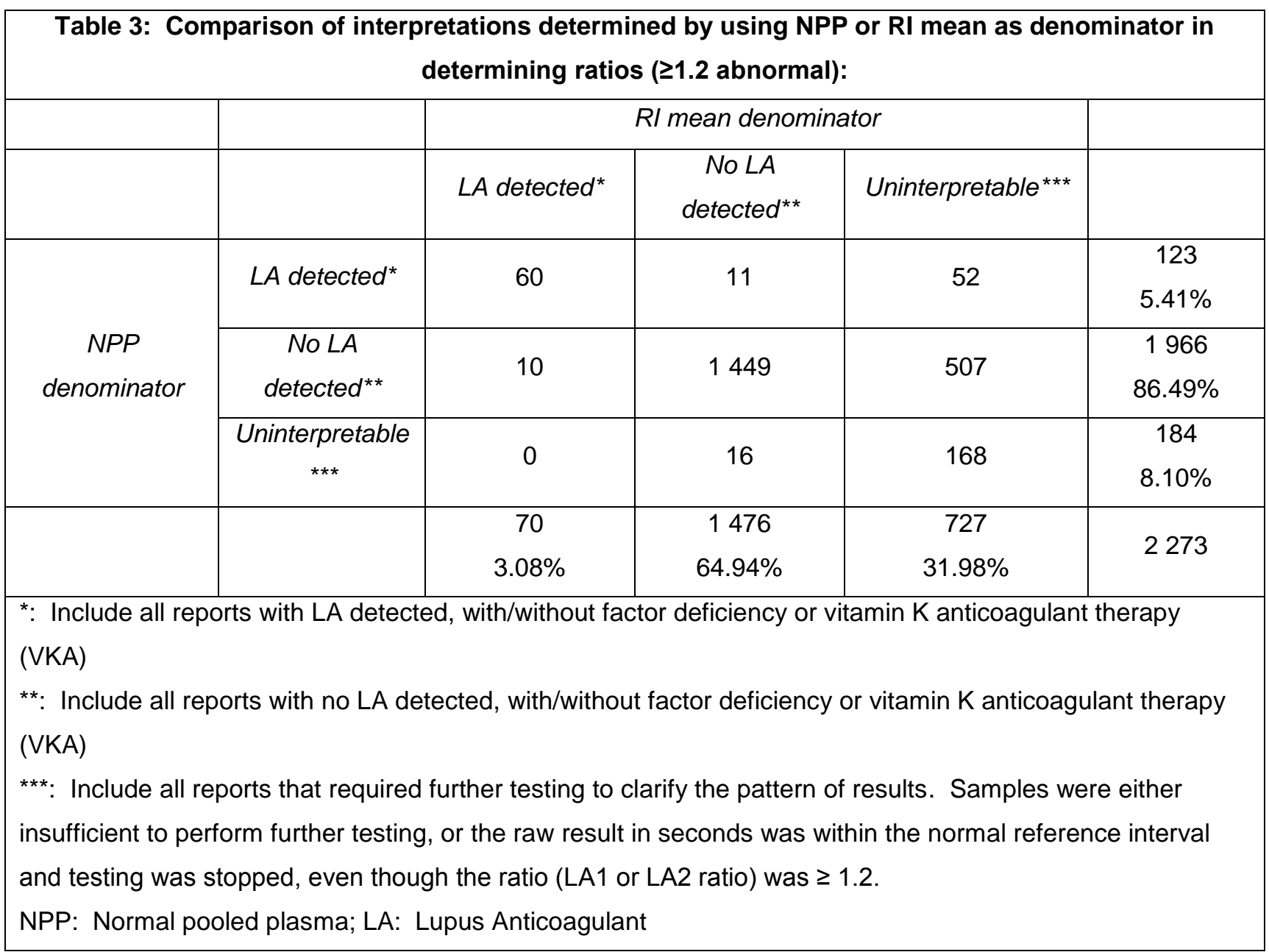

There was a statistically significant discrepancy between the interpretive comments when interpreting the clotting times vs ratios with NPP as denominator $(P$ value $<0.05)$. Using ratios, determined with NPP as denominator, and calculating normalized ratios yielded more conclusive results (LA present/not detected) than using clotting times compared to the reference interval. All guidelines advise using ratios for interpretation as ratios correct for inter-and intra-assay variation as well as operator variability.

There was a statistically significant discrepancy between the interpretive comments when interpreting ratios using NPP vs RI mean as denominator ( $P$ value $<0.05)$. Of the $727(32 \%)$ of the 2273 panels that yielded an uninterpretable report, 75 (3\%) of the 2273 required further testing, but no further testing was performed, as the ratio with the NPP as denominator was $<1.2$ and further testing was not required, as specified by the SOP. Even with this in mind, using the NPP as denominator yielded more conclusive results (LA present/not detected) that using the RI mean as interval. Table 4 demonstrates the differences in RI mean and mean of NPP clotting times. Table 5 demonstrates the mean of values that had discrepant results (LA detected vs LA not detected) in this study. These values are compared when interpreted with the mean NPP values or mean RI as 
denominator. It is evident in this study that, as demonstrated by Moore $G W^{\beta}$, that inappropriate NPP values will bias results and the interpretation thereof. This finding led us to re-evaluate our use of NPP as denominator in determining ratios. Using the RI mean will negate the significant variability in clotting times obtained from different NPP batches.

\begin{tabular}{|l|l|l|}
\hline \multicolumn{4}{|l|}{ Table 4: RI mean and mean of NPP clotting times } \\
\hline Assay & dRVVT screen (LA1) & dRVVT confirm (LA2) \\
\hline RI for clotting times (old) & $33.80-43.90$ & $28.63-36.55$ \\
\hline RI mean (s) & 42.89 & 32.59 \\
\hline $\begin{array}{l}\text { Mean clotting times for locally } \\
\text { prepared frozen NPP }\end{array}$ & 41.25 & 37.1 \\
\hline
\end{tabular}

\begin{tabular}{|l|l|l|l|}
\hline $\begin{array}{l}\text { Table 5: Comparison of RI mean vs NPP as denominator in discrepant results obtained in } \\
\text { this study }\end{array}$ & \multicolumn{1}{|c|}{ LA1 } & LA2 & NR \\
\hline \multicolumn{1}{|c|}{ Assay } & & 39.8 & \\
\hline Set 1 & 54.8 & 1.08 & 1.25 \\
\hline $\begin{array}{l}\text { Nean of clotting times positive for LA with } \\
\text { mean }\end{array}$ & 1.35 & 1.22 & 1,04 \\
\hline $\begin{array}{l}\text { Mean of ratios positive for LA with NPP as } \\
\text { denominator; negative with RI mean }\end{array}$ & 1.27 & 35.7 & \\
\hline $\begin{array}{l}\text { Set 1 values with RI mean as denominator } \\
\text { Set } 2\end{array}$ & 48.7 & 1.14 & 1.10 \\
\hline $\begin{array}{l}\text { Mean of clotting times positive for LA with } \\
\text { RI mean as denominator; negative with } \\
\text { NPP }\end{array}$ & 1.25 & 0.96 & Not indicated \\
\hline $\begin{array}{l}\text { Mean of ratios positive for LA with RI mean } \\
\text { as denominator; negative with NPP }\end{array}$ & 1.18 & & \\
\hline Set 2 with mean NPP as denominator & & \\
\hline
\end{tabular}

The SOP for dRVVT determination was reviewed and changed to clarify interpretation of dRVVT panels and the relevant interpretive comment that should be added to the report. The sequence of steps was also changed from screen, mix, confirm to screen, confirm, mix, as suggested by the CLSI 2014 guidelines. The previous sequence was still followed in 2015. This resulted in redundant mixing tests being performed, which in turn impacted on reagent cost. The number of redundant tests performed was determined. The reagent cost per assay was established and reagent cost wastage calculated. 
The reagent cost wastage was determined, and information is displayed in table 4.

\section{DISCUSSION}

The laboratory diagnosis of LA remains challenging for many laboratories, in large due to the heterogeneity of these autoantibodies and the lack of a gold standard.

The new reference interval for dRVVT had a reduced upper limit, which led to erroneous reports being authorized.

Statistically significant differences exist with all methods of interpretation evaluated in this study, but using ratios, determined with NPP as denominator, proved to yield more definitive reports (LA present/not detected) than any of the other two methods.

As mentioned in the CLSI 2014 guidelines, using the RI mean as denominator in determining ratios specify that the RI has to be verified with each lot change of reagents. ${ }^{5}$ This practice becomes costly, especially for a laboratory in a resource-restricted country as South Africa. Therefore, it will be more beneficial for our laboratory to use the NPP as denominator in determining ratios and comparing these ratios to the universal cut-off ratio of $\geq 1.2$ to determine the sequence of assays as well as the interpretive comment.

The SOP for Lupus Anticoagulant testing has been revised and amended to include a flow diagram (figure 1) to guide operators in determining which sequence of events to follow. Table 1 was included in the SOP to standardize interpretation of the ratios, using $\geq 1.2$ as abnormal, and interpretive comments added to reports. To establish adherence to the implemented corrective actions frequent audits to ensure compliance has been introduced.

Shortcomings of the study include selection bias due to the retrospective nature of the study. No background clinical information of the patients was available to assess the indication for Lupus Anticoagulant screening. An area of research that will be valuable is to assess whether any positive Lupus Anticoagulant screens were repeated at least 12 weeks later, and whether a final clinical diagnosis has been reached. This study has emphasised that quality measures cannot be introduced, maintained or enforced by a quality management system without the collective ownership and adherence of all laboratory personnel involved. 


\section{References}

1. Lim W. Antiphospholipid syndrome. Hematology Am Soc Hematol Educ Program. 2013;2013:675-680.

2. Wilson WA, Gharavi AE, Koike T, et al. International consensus statement on preliminary classification criteria for definite antiphospholipid syndrome: report of an international workshop. Arthritis and rheumatism. 1999;42(7):1309-1311.

3. Devreese KM. Antiphospholipid antibody testing and standardization. International journal of laboratory hematology. 2014;36(3):352-363.

4. Pengo V. ISTH guidelines on lupus anticoagulant testing. Thrombosis research. 2012;130 Suppl 1:S76-77.

5. Ledford-Kraemer MR, Moore GW, Bottenus R, et al. Laboratory Testing for the Lupus Anticoagulant; Approved Guideline. Vol H60-A. Wayne, PA: Clinical and Laboratory Standards Institute; 2014.

6. Keeling D, Mackie I, Moore GW, Greer IA, Greaves M, British Committee for Standards in $\mathrm{H}$. Guidelines on the investigation and management of antiphospholipid syndrome. Br J Haematol. 2012;157(1):47-58.

7. Pengo V, Tripodi A, Reber G, et al. Update of the guidelines for lupus anticoagulant detection. Subcommittee on Lupus Anticoagulant/Antiphospholipid Antibody of the Scientific and Standardisation Committee of the International Society on Thrombosis and Haemostasis. J Thromb Haemost. 2009;7(10):1737-1740.

8. Moore GW. Commonalities and contrasts in recent guidelines for lupus anticoagulant detection. International journal of laboratory hematology. 2014;36(3):364-373.

9. Tripodi A, Chantarangkul V, Cini M, et al. Variability of cut-off values for the detection of lupus anticoagulants: results of an international multicenter multiplatform study. Journal of thrombosis and haemostasis : JTH. 2017;15(6):1180-1190.

10. Favaloro EJ. Variability and diagnostic utility of antiphospholipid antibodies including lupus anticoagulants. International journal of laboratory hematology. 2013;35(3):269274. 